TRANSACTIONS OF THE

AMERICAN MATHEMATICAL SOCIETY

Volume 359, Number 4, April 2007, Pages 1817-1828

S 0002-9947(06)04091-8

Article electronically published on November 3, 2006

\title{
SOME CHARACTERIZATIONS OF SPACE-FORMS
}

\author{
STEFANO PIGOLA, MARCO RIGOLI, AND ALBERTO G. SETTI
}

Dedicated to the memory of Franca Burrone Rigoli

\begin{abstract}
Integral conditions on the traceless Ricci tensor are used to characterize Euclidean and hyperbolic spaces among complete, locally conformally flat manifolds of constant scalar curvature. The main tools are vanishing-type results for $L^{p}$-solutions of a large class of differential inequalities. Further applications of the technique are also given.
\end{abstract}

\section{INTRODUCTION}

A Riemannian manifold $(M,\langle\rangle$,$) of dimension m$ is said to be locally conformally flat if a neighborhood of each point of $M$ can be conformally immersed into the standard sphere $\mathbb{S}_{1}^{m}$. When $m \geq 4$, this is equivalent to the fact that the Weyl tensor identically vanishes. The category of locally conformally flat spaces contains the manifolds of constant sectional curvature, hence, in particular, the space-forms $\mathbb{R}^{m}, \mathbb{H}_{-k^{2}}^{m}, \mathbb{S}_{k^{2}}^{m}$. Note that, for $m \geq 3$, according to the orthogonal decomposition of the Riemann tensor into its irreducible components, a conformally flat manifold has constant sectional curvature if and only if it is Einstein, i.e., the traceless part of its Ricci tensor is identically equal to zero. As a consequence, by the H. Hopf classification theorem, the space forms are (up to isometries) the only complete, simply connected, locally conformally flat, Einstein manifolds. In this paper we investigate other possible characterizations of space forms from the conformally flat viewpoint.

In the late sixties M. Tani, [T, showed that the universal cover of a compact, orientable, $m$-dimensional, locally conformally flat Riemannian manifold $(M,\langle\rangle$, with positive Ricci curvature and constant scalar curvature $S$ is isometrically a sphere.

This result has been generalized by S.I. Goldberg, G], in the complete (nonnecessarily orientable) case under the additional assumption that, for some $\varepsilon>0$,

$$
\frac{S^{2}}{m-1}-\mid \text { Ricci }\left.\right|^{2} \geq \varepsilon>0 \quad \text { on } M
$$

(see also $[\mathrm{H}$ and the further observations in $\mathrm{CW}$ ). In fact, combining a classification theorem by S. Zhu, Z, with a celebrated global symmetry result due to

Received by the editors January 29, 2005.

2000 Mathematics Subject Classification. Primary 53C21; Secondary 35J60, 35B05.

Key words and phrases. Space forms, vanishing theorems, isolation phenomena.

(C)2006 American Mathematical Society Reverts to public domain 28 years from publication 
L. Caffarelli, B. Gidas and J. Spruck, CGS, we prove that the above characterization holds assuming merely that the left-hand side of (1.1) is strictly positive at one point.

Theorem 1.1. Let $(M,\langle\rangle$,$) be a complete, locally conformally flat Riemannian$ manifold of dimension $m \geq 3$ and with constant scalar curvature $S>0$. If

$$
\frac{S^{2}}{m-1}-\mid \text { Ricci }\left.\right|^{2} \geq 0 \quad \text { on } M
$$

and the strict inequality holds at some point, then the universal cover of $(M,\langle\rangle$, is isometrically a sphere.

We also consider analogous characterizations in the case where the scalar curvature is nonpositive.

Our first result in this direction deals with Euclidean space and can be thought of as an extension of Theorem 1.1 to the scalar flat case.

Theorem 1.2. Let $(M,\langle\rangle$,$) be a complete, simply connected, locally conformally$ flat Riemannian manifold of dimension $m \geq 3$ and zero scalar curvature. Assume that

$$
\|R i c c i\|_{L^{\frac{m}{2}}(M)}<\frac{2 \omega_{m}^{\frac{2}{m}}(m-2)^{3} \sqrt{m-1}}{m^{2} \sqrt{m}}
$$

where $\omega_{m}$ denotes the volume of the standard sphere $\mathbb{S}_{1}^{m}$. Then $(M,\langle\rangle$,$) is isometric$ to Euclidean space.

In the case of negative scalar curvature, we establish the following result.

Theorem 1.3. Let $(M,\langle\rangle$,$) be a complete, locally conformally flat Riemannian$ manifold of dimension $m \geq 4$ and constant scalar curvature $S<0$. Assume that, for some fixed $\varepsilon \geq 0$ and $p$, satisfying $m-1<m p<(m-1)(m-2)$, the following conditions hold:

$$
\text { i) }\left|\operatorname{Ricci}-\frac{S}{m}\langle,\rangle\right| \leq-\varepsilon S, \quad \text { ii) }\left|\operatorname{Ricci}-\frac{S}{m}\langle,\rangle\right| \in L^{p}(M),
$$

and furthermore

$$
\lambda_{1}^{-\Delta}(M)>\frac{p^{2}}{4} \frac{m}{m(p-1)+2}(-S)\left\{\frac{\varepsilon \sqrt{(m-1) m}+1}{(m-1)}\right\}
$$

where $\lambda_{1}^{-\Delta}(M)$ denotes the bottom of the spectrum of the (positive definite) Laplace operator $-\Delta$. Then, the universal cover of $(M,\langle\rangle$,$) is isometric to m$-dimensional hyperbolic space.

We remark the restrictions on $m$ and $p$ follow from substituting into (1.5) the values of the scalar curvature and the bottom of the spectrum of $m$-dimensional hyperbolic space, for which (1.4) i) and ii) hold with $\varepsilon=0$ and every $p>0$.

\section{Preliminaries}

The common ingredients in proving the theorems stated above are a suitable Weitzenböck formula and a refined Kato inequality that we shall briefly recall momentarily. These ingredients will be used together with a vanishing-type theorem 
for $L^{p}$-solutions of a general class of differential inequalities which depend on the value of the scalar curvature; see Proposition 3.1 below.

In what follows, we adopt, without further comment, the moving frame notation with respect to a chosen local orthonormal frame.

Let $(M,\langle\rangle$,$) be a conformally flat manifold of dimension m \geq 3$. Then, again by the decomposition of the Riemann tensor into its irreducible components, its components $R_{i j k l}$ are related to the components $R_{i j}$ of the Ricci tensor and to the scalar curvature $S$ by

$$
\begin{aligned}
R_{i j k l}=\frac{1}{m-2}\left(R_{i k} \delta_{j l}-R_{i l} \delta_{j k}+R_{j l} \delta_{i k}\right. & \left.-R_{j k} \delta_{i l}\right) \\
& -\frac{S}{(m-1)(m-2)}\left(\delta_{i k} \delta_{j l}-\delta_{i l} \delta_{j k}\right) .
\end{aligned}
$$

Assuming that $S$ is constant, from the second Bianchi identities and (2.1) we immediately obtain that Ricci is a Codazzi tensor, i.e., the following commutation rule for the coefficients of the covariant derivative DRicci hold

$$
R_{i j, k}=R_{i k, j}
$$

As a consequence, the traceless part

$$
T=R i c c i-\frac{S}{m}\langle,\rangle
$$

is again Codazzi so that, as first observed by J.P. Bourguignon, [Bo, we have the validity of the refined Kato-type inequality

$$
|D T|^{2} \geq\left.\frac{m+2}{m}|\nabla| T\right|^{2} .
$$

Here, $\nabla$ denotes the gradient operator and |.| stands for the length of the corresponding tensor. Further covariant differentiation of Ricci yields the commutation formulas

$$
R_{i j, k l}-R_{i j, l k}=R_{i t} R_{t j k l}+R_{t j} R_{t i k l} .
$$

Using (2.1), (2.2), (2.4), the fact that $S$ is constant, and the identity

$$
|T|^{2}=\mid \text { Ricci }\left.\right|^{2}-\frac{S^{2}}{m},
$$

we compute $\Delta|T|^{2}$ to get

$$
\begin{aligned}
\frac{1}{2} \Delta|T|^{2}=|D T|^{2}+\frac{m}{m-2} & t r \\
& \left(\operatorname{Ricci}^{(3)}\right) \\
& -\frac{(2 m-1) S}{(m-2)(m-1)} \mid \text { Ricci }\left.\right|^{2}+\frac{S^{3}}{(m-1)(m-2)}
\end{aligned}
$$

where $\operatorname{Ricci}^{(3)}$ is the composition of the Ricci tensor three times with itself. Expressing $\operatorname{tr}\left(\operatorname{Ricci} i^{(3)}\right)$ in terms of $T$ we obtain, with the obvious meaning of the symbols,

$$
\frac{1}{2} \Delta|T|^{2}=|D T|^{2}+\frac{m}{m-2} \operatorname{tr}\left(T^{(3)}\right)+\frac{S}{m-1}|T|^{2} .
$$

A simple algebraic lemma due to M. Okumura, O1, shows that

$$
\operatorname{tr}\left(T^{(3)}\right) \geq-\frac{m-2}{\sqrt{m(m-1)}}|T|^{3} .
$$


Inserting (2.6) into (2.5) gives

$$
\frac{1}{2} \Delta|T|^{2} \geq|D T|^{2}-\frac{m}{\sqrt{m(m-1)}}|T|^{3}+\frac{S}{m-1}|T|^{2} .
$$

Setting $u=|T|$ and using (2.3), we rewrite the above inequality in the form

$$
u \Delta u+\left(\sqrt{\frac{m}{m-1}} u-\frac{S}{m-1}\right) u^{2} \geq \frac{2}{m}|\nabla u|^{2},
$$

pointwise on $\{x \in M:|T|(x) \neq 0\}$ and weakly on all of $M$.

\section{Proofs of the Results}

We begin by giving the details of the proof of Theorem 1.1

Proof of Theorem 1.1. We note that, by a lemma of Okumura, O2], inequality (1.2) implies that Ricci $\geq 0$ on $M$. Therefore, according to [Z], the universal cover $\tilde{M}$ of $M$ is either isometric to $\mathbb{R} \times \mathbb{S}_{S /(m-1)(m-2)}^{m-1}$ or conformally equivalent to $\mathbb{R}^{m}$ or $\mathbb{S}_{1}^{m}$. Since, by assumption, inequality (1.2) is strict somewhere, the first case is excluded. On the other hand, $\tilde{M}$ cannot be conformally diffeomorphic to $\mathbb{R}^{m}$. In fact, from Theorem 8.1 in CGS we know that a Riemannian metric on $\mathbb{R}^{m}$ which is of constant, positive scalar curvature and conformally related to the canonical metric must be a spherical metric, hence incomplete. It follows that $\tilde{M}$ is conformally a sphere, hence $M$ is compact. The conclusion now follows from (the easy case of) Goldberg's argument. Namely, keeping the notations of Section 2, we have only to show that $u \equiv 0$, i.e., that $M$ is Einstein. From (2.7) we obtain

$$
\Delta u^{2} \geq b(x) u^{2} \quad \text { on } M
$$

with

$$
b(x)=\frac{2}{m-1}(S-\sqrt{m(m-1)} u(x)) .
$$

On the other hand, according to (1.2) and the fact that the strict inequality holds somewhere, we have

$$
0 \not \equiv b(x) \geq C\left\{S^{2}-(m-1)|R i c c i|^{2}(x)\right\} \geq 0,
$$

for some absolute constant $C>0$. Since $M$ is compact, applying the usual maximum principle we conclude that $b(x)$ is a positive constant and $u \equiv 0$, as desired.

The proof of Theorem 1.2 is based on the following result of independent interest.

Proposition 3.1. Let $(M,\langle\rangle$,$) be a complete manifold and assume that, for some$ $0 \leq \alpha<1$, the Sobolev-Poincaré type inequality

$$
\int_{M}|\nabla \varphi|^{2} \geq S(\alpha)^{-1}\left\{\int_{M}|\varphi|^{\frac{2}{1-\alpha}}\right\}^{1-\alpha}
$$

holds for every $\varphi \in C_{0}^{\infty}(M)$ with a positive constant $S(\alpha)>0$. Suppose that $\psi \in \operatorname{Lip}_{\text {loc }}(M)$ is a positive solution of

$$
\psi \Delta \psi+q(x) \psi^{2} \geq A|\nabla \psi|^{2} \quad \text { (weakly) on } M
$$

satisfying

$$
\int_{B_{r}}|\psi|^{\sigma}=o\left(r^{2}\right) \text { as } r \rightarrow+\infty
$$


with $A \in \mathbb{R}, A+\sigma-1>0, \sigma \neq 0$, and $q(x) \in C^{0}(M)$. Then

$$
\left\|q_{+}(x)\right\|_{L^{\frac{1}{\alpha}(M)}} \geq 4 S(\alpha)^{-1} \frac{A+\sigma-1}{\sigma^{2}} .
$$

Furthermore, if $\psi$ is assumed to be nonnegative and not identically zero, then (3.5) holds under the further assumption that $\sigma>0$.

We remark for future use that in the case where $\alpha=0$, then the constant $S(0)^{-1}$ coincides with the bottom of the $L^{2}$-spectrum of the Laplacian, $\lambda_{1}^{-\Delta}(M)$.

Proof. We consider the case where $\psi$ is only assumed to be nonnegative, and $0<$ $\sigma<2$, the other cases being easier.

To begin, recall that, by (3.3), for every test function $0 \leq \rho \in H_{c}^{1}$ we have

$$
\int q_{+} \psi^{2} \rho \geq \int \psi\langle\nabla \psi, \nabla \rho\rangle+(A+1) \rho|\nabla \psi|^{2} .
$$

Let $\phi=\phi_{r} \in C_{0}^{\infty}(M)$ be a family of cut-off functions satisfying

$$
\phi_{r} \equiv 1 \text { on } B_{r} ; \quad \phi_{r} \equiv 0 \text { off } B_{2 r} ; \quad\left|\nabla \phi_{r}\right| \leq \frac{4}{r} \text { on } M .
$$

and apply the above inequality to the test function $\left(\psi^{2}+\eta\right)^{\frac{\sigma-2}{2}} \phi^{2}$ to obtain, after some manipulation,

$$
\begin{aligned}
& \int q_{+} \phi^{2}\left(\psi^{2}+\eta\right)^{\frac{\sigma}{2}} \frac{\psi^{2}}{\psi^{2}+\eta} \geq 2 \int\left(\psi^{2}+\eta\right)^{\frac{\sigma-2}{2}} \psi \phi\langle\nabla \phi, \nabla \psi\rangle \\
&+\int\left(\psi^{2}+\eta\right)^{\frac{\sigma-2}{2}} \phi^{2}|\nabla \psi|^{2}\left\{A+1+(\sigma-2) \frac{\psi^{2}}{\psi^{2}+\eta}\right\} .
\end{aligned}
$$

We use the fact that

$$
0 \leq \frac{\psi^{2}}{\psi^{2}+\eta} \leq 1
$$

and that $\sigma-2<0$ to estimate the LHS from above, and the second integral on the RHS from below. Also, by Young's inequality and (3.7), for every $\epsilon>0$, the first integral on the RHS is estimated from below by

$$
-\frac{1}{\epsilon} \int\left(\psi^{2}+\eta\right)^{\frac{\sigma}{2}}|\nabla \psi|^{2}-\epsilon \int\left(\psi^{2}+\eta\right)^{\frac{\sigma-2}{2}} .
$$

Inserting the resulting inequalities and rearranging, we conclude that

$$
\begin{aligned}
\int q_{+} \phi^{2}\left(\psi^{2}+\eta\right)^{\frac{\sigma}{2}}+\frac{1}{\epsilon} \int\left(\psi^{2}+\eta\right)^{\frac{\sigma}{2}}|\nabla \psi|^{2} & \\
& \geq(A+\sigma-1-\epsilon) \int \phi^{2}\left(\psi^{2}+\eta\right)^{\frac{\sigma-2}{2}}|\nabla \psi|^{2} .
\end{aligned}
$$

Fix $\epsilon>0$ small enough that $A+\sigma-1-\epsilon>0$. As $\eta \searrow 0$, by dominated convergence, the LHS converges to

$$
\int q_{+} \phi^{2} \psi \sigma+\frac{1}{\epsilon} \int \psi^{\sigma}|\nabla \psi|^{2},
$$

while, since $\sigma-2<0$, by monotone convergence, the RHS converges to

$$
(A+\sigma-1-\epsilon) \int \phi^{2} \psi^{\sigma-2}|\nabla \psi|^{2} .
$$


We may therefore conclude that the integral

$$
\int \phi^{2}\left(\psi^{2}+\eta\right)^{\frac{\sigma-2}{2}}|\nabla \psi|^{2}
$$

is uniformly bounded, and that

$$
\begin{aligned}
\int q_{+}(x) \phi^{2} \psi^{\sigma}+\frac{1}{\varepsilon} \int \psi^{\sigma}|\nabla \phi|^{2} & \\
& \geq(A+\sigma-1-\varepsilon) \int \varphi^{2} \psi^{\sigma-2}|\nabla \psi|^{2} .
\end{aligned}
$$

Since, as $\eta \searrow 0,\left(\psi^{2}+\eta\right)^{\frac{\sigma}{4}} \phi \rightarrow \psi^{\frac{\sigma}{2}} \phi$ in $L^{2}$, and, by Young's inequality and (3.7),

$$
\begin{aligned}
\left|\nabla\left(\psi^{2}+\eta\right)^{\frac{\sigma}{2}} \phi\right|^{2} \leq\left(1+\frac{1}{\delta}\right)\left(\psi^{2}+\eta\right)^{\frac{\sigma}{2}}|\nabla \phi|^{2} & \\
& +(1+\delta) \frac{\sigma^{2}}{4}\left(\psi^{2}+\eta\right)^{\frac{\sigma-2}{2}} \phi^{2}|\nabla \psi|^{2},
\end{aligned}
$$

for every $\delta>0$, we deduce that $\left(\psi^{2}+\eta\right)^{\frac{\sigma}{4}} \phi$ is uniformly bounded in $H^{1}$, and therefore (see, e.g., Lemma 6.2 in $[\mathbf{F}]) \psi^{\frac{\sigma}{2}} \phi \in H_{0}^{1}(M)$ and $\nabla\left(\left(\psi^{2}+\eta\right)^{\frac{\sigma}{4}} \phi\right) \rightarrow$ $\nabla\left(\psi^{\frac{\sigma}{2}} \phi\right)$ weakly in $L^{2}$.

By the Poincaré-Sobolev inequality and (3.9), we estimate

$$
\begin{aligned}
& S(\alpha)^{-1}\left\{\int\left(\psi^{\frac{\sigma}{2}} \phi\right)^{\frac{2}{1-\alpha}}\right\}^{1-\alpha} \leq \int\left|\nabla\left(\psi^{\frac{\sigma}{2}} \phi\right)\right|^{2} \leq \liminf _{\eta \rightarrow 0+} \int\left|\nabla\left(\left(\psi^{2}+\eta\right)^{\frac{\sigma}{4}} \phi\right)\right|^{2} \\
& \leq(1+\delta) \frac{\sigma^{2}}{4} \int \psi^{\sigma-2} \phi^{2}|\nabla \psi|^{2}+\left(1+\frac{1}{\delta}\right) \int \psi^{\sigma}|\nabla \phi|^{2} \\
& \leq \frac{\sigma^{2}}{4} \frac{1+\delta}{A+\sigma-1-\epsilon}\left\{\int \psi^{\frac{\sigma}{2}} \phi^{2}+\frac{1}{\epsilon} \int \psi^{\sigma}|\nabla \psi|^{2}\right\}+\left(1+\frac{1}{\delta}\right) \int \psi^{\sigma}|\nabla \psi|^{2} .
\end{aligned}
$$

Using Hölder's inequality in the first integral,

$$
\int q_{+} \psi^{\sigma} \phi^{2} \leq\left\|q_{+}\right\|_{L^{1 / \alpha}}\left\{\int\left(\psi^{\frac{\sigma}{2}} \phi\right)^{\frac{2}{1-\alpha}}\right\}^{1-\alpha},
$$

and rearranging, we finally obtain

$$
\begin{aligned}
& \left\{S(\alpha)^{-1}-\frac{\sigma^{2}}{4} \frac{1+\delta}{A+\sigma-1-\epsilon}\left\|q_{+}\right\|_{L^{1 / \alpha}}\right\}\left\{\int\left(\psi^{\frac{\sigma}{2}} \phi\right)^{\frac{2}{1-\alpha}}\right\}^{1-\alpha} \\
& \leq\left\{\frac{\sigma^{2}}{4 \epsilon} \frac{1+\delta}{A+\sigma-1-\epsilon}+\left(1+\frac{1}{\delta}\right)\right\} \frac{C}{R^{2}} \int_{B_{2 R}} \psi^{\sigma} .
\end{aligned}
$$

To conclude, assume that (3.5) does not hold. Then, we may choose $\delta$ and $\epsilon$ small enough such that the coefficient on the LHS is positive. Since $\psi$ does not vanish identically, there exists $R$ such that, for every $r>R$ the integral is strictly positive. The required contradiction follows by noting that, according to (3.4), the right-hand side tends to zero as $r \rightarrow+\infty$.

Proof of Theorem 1.2. We keep the notation of the introduction and we observe that $u=\mid$ Ricci $\mid$ while (2.7) becomes

$$
u \Delta u+q(x) u^{2} \geq \frac{2}{m}|\nabla u|^{2}
$$


with

$$
q(x)=\sqrt{\frac{m}{m-1}} u(x) .
$$

Again, we have to show that $u \equiv 0$. To this end we reason by contradiction. Since $(M,\langle\rangle$,$) is simply connected and locally conformally flat, by a result of \mathrm{N}$. Kuiper, $[\mathrm{K}]$, there is a (global) conformal immersion (in fact embedding) of $M$ into the standard sphere $\mathbb{S}_{1}^{m}$. It follows by a result of R. Schoen and S.T. Yau, $\underline{\mathrm{SY}}$, that the Yamabe invariant $Q(M)$ of $M$ satisfies

$$
Q(M)=Q\left(\mathbb{S}_{1}^{m}\right)=\frac{m(m-2) \omega_{m}^{\frac{2}{m}}}{4} .
$$

Thus, since $S=0$, we have

$$
\int|\nabla \varphi|^{2} \geq Q\left(\mathbb{S}_{1}^{m}\right)\left(\int \varphi^{\frac{2 m}{m-2}}\right)^{\frac{m-2}{m}}, \text { for each } \varphi \in C_{0}^{\infty}(M) \backslash\{0\},
$$

which is the Sobolev inequality (3.2) with $\alpha=\frac{2}{m}$ and $S(\alpha)=Q\left(\mathbb{S}_{1}^{m}\right)^{-1}$. According to (3.10), conditions (3.3) and (3.4) are satisfied with $A=\frac{2}{m}$ and $\sigma=\frac{m}{2}$. By Proposition 3.1 and (3.11) we conclude

$$
\|\operatorname{Ricci}\|_{L^{\frac{m}{2}}(M)} \geq \frac{2 \omega_{m}^{\frac{2}{m}}(m-2)^{3} \sqrt{m-1}}{m^{2} \sqrt{m}}
$$

contradicting (1.3).

The proof of Theorem 1.3 is similar.

Proof of Theorem 1.3. As in the previous arguments, the key point is to show that $u$, i.e., the length of the traceless Ricci tensor, vanishes identically.

Using (1.4) i) into (2.7) we see that $u$ satisfies

$$
u \Delta u-S\left(\varepsilon \sqrt{\frac{m}{m-1}}+\frac{1}{m-1}\right) u^{2} \geq \frac{2}{m}|\nabla u|^{2},
$$

and $u \in L^{p}(M)$ by assumption (1.4) ii). If $u$ were not identically zero, an application of Proposition 3.1, with the choices $A=\frac{2}{m}$ and $q(x)=-S\left(\varepsilon \sqrt{\frac{m}{m-1}}+\frac{1}{m-1}\right)$, would contradict (1.5). Thus, $u \equiv 0$ as required.

\section{Further applications of Proposition 3.1}

Theorem 1.2 can be considered as an isolation phenomenon for the Ricci tensor of a scalar flat, conformally flat manifold. It is based on the vanishing-type result in the presence of a Sobolev inequality stated in Proposition 3.1 above. We point out that the same technique can be used to obtain isolation phenomena in a number of different settings. By way of example, we investigate the topology at infinity of complete submanifolds of a Cartan-Hadamard space, i.e., a complete, simply connected manifold with nonpositive sectional curvature and show how the technique introduced above allows us to give a quantitative improvement on results obtained in Ca1] and more recently in PRS.

We recall that, given a compact set $K \subset M$, an end $E$ of $M$ corresponding to $K$ is an unbounded, connected component of the set $M \backslash K$. We say that $M$ has finitely many ends if there exists a positive integer $b$ such that, for any compact set $K \subset M$, the number of ends corresponding to $K$ is bounded by $b$. In this case we 
can obviously find an integer $b_{0} \leq b$ and a compact set $K_{0}$ in such a way that $M$ has precisely $b_{0}$ ends for every compact set containing $K_{0}$. We say that $b_{0}$ is the number of ends of $M$.

Now, let us consider an isometric immersion $f:\left(M^{m},\langle\rangle,\right) \rightarrow\left(N^{n},(),\right)$ where $N$ is always assumed to be Cartan-Hadamard. Denote by II and $H$ the second fundamental tensor and the mean curvature vector field of $f$. We know from work of D. Hoffman and J. Spruck, [HS], that the following $L^{1}$-Sobolev inequality holds:

$$
S_{1}(m)^{-1}\left(\int_{M}|u|^{\frac{m}{m-1}}\right)^{\frac{m-1}{m}} \leq \int_{M}(|\nabla u|+|H||u|) \forall u \in H_{0}^{1}(M)
$$

with

$$
S_{1}(m)=\frac{\pi 2^{m-1}}{\omega_{m}^{\frac{1}{m}}} \frac{(m+1)^{1+\frac{1}{m}}}{m-1},
$$

$\omega_{m}$ being the volume of the unit ball of $\mathbb{R}^{m}$. Applying (4.1) to $u=|v|^{\frac{2(m-1)}{m-2}}$ with $v \in C_{0}^{\infty}(M)$ gives, after some manipulation,

$$
\begin{aligned}
&\left(\frac{(m-2)}{2(m-1) S_{1}(m)}\right)^{2}\left(\int_{M}|v|^{\frac{2(m-1)}{m-2}}\right)^{\frac{m-2}{m}} \\
& \leq\left\{\left(\int_{M}|\nabla v|^{2}\right)^{\frac{1}{2}}+\left(\int_{M}|H|^{2}|v|^{2}\right)^{\frac{1}{2}}\right\}^{2} .
\end{aligned}
$$

Expanding the square on the right-hand side and applying the inequality $2 a b \leq$ $\varepsilon^{2} a^{2}+\varepsilon^{-2} b^{2}$ with $\varepsilon>0$, we finally obtain the $L^{2}$-Sobolev inequality

$$
S_{2}(m, \varepsilon)^{-1}\left(\int_{M}|v|^{\frac{2 m}{m-2}}\right)^{\frac{m-2}{m}} \leq \int_{M}|\nabla v|^{2}+\varepsilon^{2} \int_{M}|H|^{2} v^{2}
$$

where

$$
S(m, \varepsilon)=\left(\frac{2 \sqrt{1+\varepsilon^{-2}}(m-1)}{(m-2) S_{1}(m)^{-1}}\right)^{2} .
$$

We observe that if $f$ is a minimal immersion, then the best $L^{2}$-Sobolev constant in (4.4) is achieved by choosing $\varepsilon=+\infty$. In this situation, we set $S_{2}(m)=$ $S_{2}(m,+\infty)$.

Combining Proposition 3.1 with some careful computations of $[\mathrm{B}$, we obtain the following isolation phenomenon for minimal submanifolds of the Euclidean space, first pointed out by M. Anderson, $\mathrm{A}$, and later quantified by P. Berard, $\mathrm{B}$.

Theorem 4.1. Let $f:\left(M^{m},\langle\rangle,\right) \rightarrow \mathbb{R}^{n}$ be a complete, minimal, immersed submanifold of dimension $m \geq 3$ whose second fundamental tensor II satisfies

$$
\left(\int_{M}|\mathrm{II}|^{m}\right)^{\frac{2}{m}}<4 S_{2}(m)^{-1} \frac{\frac{2}{(m+2)(n-m)-2}+m-1}{\left(2-\frac{1}{n-m}\right) m^{2}} .
$$

Then $f$ is totally geodesic. 
Proof. From Proposition I.2 of [B] we know that the function $\psi=|\mathrm{II}|$ is a (weak) solution of

$$
\psi \Delta \psi+\left(2-\frac{1}{n-m}\right)|\mathrm{II}|^{2} \psi^{2} \geq \frac{2}{(m+2)(n-m)-2}|d \psi|^{2} .
$$

Moreover, by (4.5), $\psi \in L^{m}(M)$. To conlcude that $\psi \equiv 0$ simply apply Proposition 3.1 with the choices $a(x)=\left(2-\frac{1}{n-m}\right)|\mathrm{II}|^{2}, \sigma=m, A=\frac{2}{(m+2)(n-m)-2}$.

L. Ni, [N], observed that the integral bound (4.5) can be relaxed in such a way that the minimal submanifold still has a simple topology at infinity, i.e., it has only one end. We are going to extend this result to nonnecessarily minimal submanifolds of a Cartan-Hadamard space.

To this end, we recall that the $L^{1}$-Sobolev inequality (4.1) has a classical application to the volume growth of the underlying manifold $M$. We shall combine this fact together with an integrability condition on $|H|$ to get a uniform lower bound on the growth of geodesic balls contained in a neighborhood of infinity. Indeed, (4.1) implies that, for every geodesic ball $B_{r}(p)$ of $M$,

$$
S_{1}(m)^{-1}\left(\operatorname{vol} B_{r}(p)\right)^{\frac{m-1}{m}} \leq\left(\operatorname{vol} \partial B_{r}(p)+\int_{B_{r}(p)}|H|\right) .
$$

If we assume

$$
\int_{M}|H|^{m}<+\infty
$$

then we can chose a compact set $K \subset M$ so large that

$$
\left(\int_{M-K}|H|^{m}\right)^{1 / m}<\frac{S_{1}(m)^{-1}}{2} .
$$

Whence, using Hölder inequality, the term involving the mean curvature can be absorbed into the left-hand side of the above and we get, for every $B_{r}(p) \subset M-K$,

$$
\frac{S_{1}(m)^{-1}}{2}\left(\operatorname{vol} B_{r}(p)\right)^{\frac{m-1}{m}} \leq \operatorname{vol} \partial B_{r}(p) .
$$

Integrating this inequality gives

$$
\operatorname{vol} B_{r}(p) \geq C r^{m},
$$

for every $B_{r}(p) \subset M-K$ and for some constant $C>0$ independent of $p$.

According to a nice argument of H. Cao, Y. Shen and S. Zhu (see Lemma 2 in [CSZ]), the validity of the (standard) $L^{2}$-Sobolev inequality on $M$,

$$
c_{m}^{-1}\left(\int_{M}|v|^{\frac{2 m}{m-2}}\right)^{\frac{m-2}{m}} \leq \int_{M}|\nabla v|^{2}, v \in C_{0}^{\infty}(M),
$$

together with a "uniform" volume growth condition in a neighborhood of infinity (like that expressed in (4.7)) can be used to relate the number of ends of $M$ to the presence of nonconstant, bounded harmonic functions with finite energy. Now, the above considerations show that $M$ inequality (4.8) holds outside a large compact set in $M$. Its validity on all of $M$ now follows from work of G. Carron, Ca1], Proposition 2.5.

Summarizing, we have the following version of Lemma 1 and Lemma 2 in [CSZ]. 
Lemma 4.2. Let $f:\left(M^{m},\langle\rangle,\right) \rightarrow\left(N^{n},(),\right)$ be an isometric immersion of the complete manifold $M$ of dimension $m \geq 3$ into the Cartan-Hadamard manifold $N$. Denote by $H$ the mean curvature vector field of $f$ and assume the validity of (4.6). Then, each end of $M$ has infinite volume. Moreover, if there are at least two ends, then $M$ supports a nonconstant, bounded, harmonic function with finite energy.

We are now in the position to prove our isolation phenomenon for the number of ends of submanifolds of a Cartan-Hadamard space with controlled second fundamental tensor.

Theorem 4.3. Let $f:\left(M^{m},\langle\rangle,\right) \rightarrow\left(N^{n},(),\right)$ be an isometric immersion of the complete manifold $M$ of dimension $m \geq 3$ into the Cartan-Hadamard manifold $N$ whose sectional curvature (along $f$ ) satisfies

$$
(0 \geq){ }^{N} \operatorname{Sec}_{f(x)} \geq-{ }^{N} R(x)
$$

for some function ${ }^{N} R \in C^{0}(M)$. Denote by $H$ and II respectively the mean curvature vector field and the second fundamental tensor of $f$. Assume that, for some $\varepsilon>0$

$$
\left\|(m-1){ }^{N} R(x)+|\mathrm{II}|(|\mathrm{II}|+m|H|)(x)\right\|_{L^{\frac{m}{2}}}<\frac{m}{m-1} S_{2}(m, \varepsilon)^{-1}
$$

where $S_{2}(m, \varepsilon)$ represents the $L^{2}$-Sobolev constant defined in (4.4). Then $M$ has only one end.

Proof. We define $q \in C^{0}(M)$ by setting

$$
q(x)=(m-1){ }^{N} R(x)+|\mathrm{II}|(|\mathrm{II}|+m|H|)(x),
$$

and we note that, by Gauss equations,

$$
{ }^{M} \text { Ricci } \geq-q(x) .
$$

According to Lemma 4.2 we have to show that $M$ does not support any nonconstant, bounded, harmonic function with finite Dirichlet integral. By contradiction, we suppose that such a function $u$ exists and we set $\psi=|\nabla u|$. Then, by the Weitzenböck-Bochner formula for harmonic functions we have that $\psi \in L^{2}(M)$ is a nonnull solution of

$$
\psi \Delta \psi+q(x) \psi^{2} \geq \frac{1}{(m-1)}|\nabla \psi|^{2} .
$$

Applying Proposition 3.1 with $A=\frac{1}{m-1}$ and $\sigma=2$ we immediately contradict (4.10).

Remark 4.4. Theorem 4.3 compares with Theorem 2.13 in [PRS] where the quantitative isolation phenomenon is obtained assuming that

$$
\|q(x)\|_{L^{\frac{m}{2}}} \leq S_{2}(m, \varepsilon)^{-1} .
$$

The argument there relies on a vanishing result, Theorem 1.4, which depends on the assumption that the bottom of the spectrum of the Schrödinger operator $-\Delta-q$ is nonnegative. This in turn is obtained by combining the integral bound (4.12) and the Sobolev inequality. By contrast, the argument in Proposition 3.1 uses the Sobolev inequality in a more direct way, and allows us to improve the constant. Similar isolation phenomena were previously obtained, with a different technique, by Carron (see Ca1], Ca2]), where, however, the constant in the integral bound is not explicit. 
Our next result is a further application of Proposition 3.1 and it implies a vanishing result for harmonic forms in $L^{2}$.

Theorem 4.5. Let $(M,\langle\rangle$,$) be a complete Riemannian manifold supporting the$ Poincaré-Sobolev inequality (3.2) for some $0 \leq \alpha<1$, and assume that

$$
\text { Ricci } \geq-\rho(x)
$$

for some continuous function $\rho$ satisfying

$$
\left\|\rho_{+}\right\|_{L^{1 / \alpha}}<\frac{4}{p^{2}} \frac{(m-1)(p-1)+1}{m-1} S(\alpha)^{-1},
$$

for some $p>(m-2) /(m-1)$. If $\omega$ is a closed and co-closed 1-form satisfying

$$
\int_{B_{r}}|\omega|^{p}=o\left(r^{2}\right)
$$

then $\omega \equiv 0$.

The proof follows by noting that the norm of a closed and co-closed 1-form satisfies the differential inequality (4.11) and applying Proposition 3.1. Noting that a harmonic $L^{2}$ form is automatically closed and co-closed, we recover the vanishing results of $\mathrm{P}$. Li and J. Wang, $\mathrm{LW}$, Theorem 4.2, for manifolds with a positive spectral gap. In the case of $L^{p}$ harmonic 1-forms, which are not necessarily closed and co-closed, and therefore do not satisfy a refined Kato inequality, one has a vanishing result provided the right-hand side of (4.13) is replaced by $4(p-1) S(\alpha)^{-1} / p^{2}$.

Similar results can be given for $L^{p}$ harmonic forms of any degree, or for harmonic maps with $L^{p}$ energy density, provided one uses the appropriate Weitzenböck formula. For instance, we have the following.

Theorem 4.6. Let $(M,\langle\rangle$,$) be an m-dimensional complete Riemannian manifold,$ supporting the Poincaré-Sobolev inequality (3.2) for some $0 \leq \alpha<1$, and assume that

$$
\text { Ricci } \geq-\rho(x) \text {. }
$$

Let $f: M \rightarrow N$ be a harmonic map into an $n$-dimensional Riemannian manifold $N$. If $|d f| \in L^{p}(M)$, for some $p>\frac{m-2}{m-1}$ and

$$
\left\|\rho_{+}\right\|_{L^{1 / \alpha}}<\frac{4}{p^{2}} \frac{(m-1)(p-1)+1}{(m-1)} S(\alpha)^{-1},
$$

then $f$ is constant.

\section{REFERENCES}

[A] M. Anderson, The compactification of a minimal submanifold in Euclidean space by the Gauss map. Preprint.

[B] P. Bérard, Remarques sur l'équation de J. Simons. Differential geometry, 47-57, Pitman Monogr. Surveys Pure Appl. Math., 52, Longman Sci. Tech., Harlow, 1991. MR1173032 (93g:53082)

[Bo] J.P. Bourguignon, The magic of Weitzenböck formulas, Variational Methods Paris (1998), 251-271. Progess in Nonlinear Differential Equations and Applications IV, Birkauser, 1990. MR.1205158 (94a:58181)

[Ca1] G. Carron, Une suite exacte en $L^{2}$-cohomologie. Duke Math. J. 95 (1998), 343-372. MR.1652017 (99g:58115)

[Ca2] G. Carron, L'L -Cohomologie et inégalités de Sobolev, Math. Ann. 314 (1999), 614-639. MR:1709104 (2000f:53045) 
[CGS] L. Caffarelli, B. Gidas, J. Spruck, Asymptotic symmetry and local behavior of semilinear elliptic equations with critical Sobolev growth. Comm. Pure Appl. Math. 42 (1989), 271297. MR0982351 (90c:35075)

[CSZ] H.-D. Cao, Y. Shen, S. Zhu, The structure of stable minimal hypersurfaces in $\mathbb{R}^{m+1}$. Math. Res. Lett. 4 (1997), 637-644. MR1484695 (99a:53037)

[CW] Q.M. Cheng, B.Q. Wu, The generalized maximum principle and conformally flat spaces, Northeastern Math. J. 8 (1992), 54-56. MR.1164748 (93g:53052)

[F] A. Friedman, Partial Differential Equations, Holt, Rinehart and Winston, Inc., New York 1970. MR0445088 (56:3433)

[G] S.I. Goldberg, An application of Yau's maximum principle to conformally flat spaces. Proc. Amer. Math. Soc. 79 (1980), 268-270. MR0565352 (81j:53043)

$[\mathrm{H}] \quad$ T. Hasanis, Conformally flat spaces and a pinching problem for the Ricci tensor. Proc. Amer. Math. Soc. 86 (1982), 312-315. MR0667296 (84k:53043)

[HS] D. Hoffman, J. Spruck, Sobolev and isoperimetric inequalities for Riemannian submanifolds. Comm. Pure Appl. Math. 27 (1974), 715-727. MR0365424(51:1676)

[K] N. Kuiper, On conformally flat spaces in the large, Annals of Math. 50 (1949), 916-924. MR0031310(11:133b)

[LW] P. Li and J. Wang, Complete manifolds with positive spectrum, J. Diff. Geom. 58 (2001), 501-534. MR1906784 (2003e:58046)

[N] L. Ni, Gap theorems for minimal submanifolds in $\mathbb{R}^{n+1}$. Comm. Anal. Geom. 9 (2001), 641-656. MR1895136 (2002m:53097)

[O1] M. Okumura, Hypersurfaces and a pinching problem on the second fundamental tensor. Amer. J. Math. 96 (1974), 207-213. MR0353216 (50:5701)

[O2] M. Okumura, Submanifolds and a pinching problem on the second fundamental tensors. Trans. Amer. Math. Soc. 178 (1973), 285-291. MR0317246 (47:5793)

[PRS] S. Pigola, M. Rigoli, A.G. Setti, Vanishing theorems on Riemannian manifolds and geometric applications. J. Funct. Anal. 229 (2005), 424-461. MR2182595

[SY] R. Schoen, S.T. Yau, Lectures on differential geometry. Conference Proceedings and Lecture Notes in Geometry and Topology, I. International Press, Cambridge, MA, 1994. MR.1333601 (97d:53001)

[T] M. Tani, On a conformally flat Riemannian space with positive Ricci curvature. Tôhoku Math. J. (2) 19 (1967), 227-231. MR0220213 (36:3279)

[Z] S. Zhu, The classification of complete conformally flat manifolds of nonnegative Ricci curvature, Pacific J. Math. 163 (1994), 189-199. MR1256184 (95d:53045)

Dipartimento di Fisica e Matematica, Università dell'insubria - Como, via Valleggio 11, I-22100 Como, Italy

E-mail address: stefano.pigola@uninsubria.it

Dipartimento di Matematica, Università di Milano, via Saldini 50, I-20133 Milano, ITALY

E-mail address: rigoli@mat.unimi.it

Dipartimento di Fisica e Matematica, Università dell'Insubria - Como, via Valleggio 11, I-22100 Como, Italy

E-mail address: alberto.setti@uninsubria.it 